\title{
The GHS and Other Correlation Inequalities for a Class of Even Ferromagnets
}

\author{
Richard S. Ellis * \\ Department of Mathematics, Northwestern University, Evanston, Illinois, USA \\ James L. Monroe $\star \star$ \\ Department of Physics, Northwestern University, Evanston, Illinois, USA \\ Charles M. Newman $\star \star \star ~$ \\ Department of Mathematics, Indiana University, Bloomington, Indiana, USA
}

\begin{abstract}
We prove the GHS inequality for families of random variables which arise in certain ferromagnetic models of statistical mechanics and quantum field theory. These include spin $-1 / 2$ Ising models, $\varphi^{4}$ field theories, and other continuous spin models. The proofs are based on the properties of a class $\mathscr{G}_{-}$ of probability measures which contains all measures of the form const $\exp (-V(x)) d x$, where $V$ is even and continuously differentiable and $d V / d x$ is convex on $[0, \infty)$. A new proof of the GKS inequalities using similar ideas is also given.
\end{abstract}

\section{Introduction}

We consider models of general even ferromagnets with pair interactions in a positive external magnetic field. Such a model is defined by a finite family of real-valued random variables $\left\{X_{i} ; i=1, \ldots, N\right\}$, whose joint probability distribution $\tau_{h_{1} \ldots h_{N}}$ on $\mathbb{R}^{N}$ has the form

$$
\tau_{h_{1} \ldots h_{N}}\left(d x_{1}, \ldots, d x_{N}\right)=\left(1 / Z\left(h_{1}, \ldots, h_{N}\right)\right) \exp \left(-H\left(x_{1}, \ldots, x_{N}\right)\right) \prod_{i=1}^{N} \varrho_{i}\left(d x_{i}\right) .
$$

$H\left(x_{1}, \ldots, x_{N}\right)$ is the Hamiltonian defined by

$$
H\left(x_{1}, \ldots, x_{N}\right)=-\sum_{1 \leqq i \leqq j \leqq N} J_{i j} x_{i} x_{j}-\sum_{1 \leqq i \leqq N} h_{i} x_{i},
$$

and $Z\left(h_{1}, \ldots, h_{N}\right)$ is the partition function defined by

$$
Z\left(h_{1}, \ldots, h_{N}\right)=\int_{\mathbb{R}^{N}} \ldots \int \exp \left(-H\left(x_{1}, \ldots, x_{N}\right)\right) \prod_{i=1}^{N} \varrho_{i}\left(d x_{i}\right) .
$$

The indices $i$ and $j$ label atomic sites in a lattice $\Lambda=\{1, \ldots, N\}$ of $N$ sites. $X_{i}$ denotes the spin of the $i^{\prime}$ th atom, $J_{i j} \geqq 0$ the ferromagnetic interaction strength between $X_{i}$ and $X_{j}$, and $h_{i} \geqq 0$ the non-negative external magnetic field strength at the

\footnotetext{
* Supported in part by National Science Foundation Grant MPS 71-02838 A 04.

Current address: Department of Mathematics, University of Massachusetts, Amherst, MA 01002, USA

$\star \star$ Supported by National Science Foundation Grant MPS 74-24696.

Current address: Department of Chemistry, State University of New York, Stony Brook, N.Y. 11794, USA

$\star \star \star$ Supported in part by National Science Foundation Grant MPS 74-04870. Israel

Current address (until June, 1976): Departments of Mathematics and Physics, Technion, Haifa,
} 
$i$ th site. The $\varrho_{i}$ are measures belonging to $\mathscr{E}$, which is the set of all even probability measures $\varrho$ satisfying

$$
\int \exp \left(k x^{2}\right) \varrho(d x)<\infty \text { for some } k>0 .
$$

The choice of each $\varrho_{i}$ as the Bernoulli measure

$$
b(d x)=\frac{1}{2}(\delta(x-1)+\delta(x+1))
$$

gives a classical spin $-1 / 2$ Ising model [30, Chapter 5].

We shall assume that the numbers $J_{i j}$ are so small that the integral in (1.3) converges for all $h_{i} \geqq 0$. Unless noted otherwise, the inequalities we discuss are to hold for all $h_{i} \geqq 0$ and all $J_{i j} \geqq 0$ subject only to this restriction.

Our main focus in this paper is on the Griffiths-Hurst-Sherman (GHS) inequality, which states that

$$
\begin{aligned}
0 \geqq \partial^{3} / \partial h_{i} \partial h_{j} \partial h_{k} \ln Z\left(h_{1}, \ldots, h_{N}\right) \\
=E\left\{\left(X_{i}-E\left\{X_{i}\right\}\right)\left(X_{j}-E\left\{X_{j}\right\}\right)\left(X_{k}-E\left\{X_{k}\right\}\right)\right\} \\
=E\left\{X_{i} X_{j} X_{k}\right\}-E\left\{X_{i}\right\} E\left\{X_{j} X_{k}\right\}-E\left\{X_{j}\right\} E\left\{X_{i} X_{k}\right\} \\
\left.\quad-E\left\{X_{k}\right\} E\left\{X_{i} X_{j}\right\}+2 E\left\{X_{i}\right\} E X_{j}\right\} E\left\{X_{k}\right\}
\end{aligned}
$$

for all (not necessarily distinct) sites $i, j, k \in \Lambda$. We discuss several implications of (1.4) below. The GHS inequality was first shown to hold for spin-1/2 Ising models [11]. Also, one can prove the GHS inequality indirectly for ferromagnetic families of random variables. These are systems which can be built out of spin $-1 / 2$ Ising models in a suitable way $[18, \S 4]$; examples are given after the statement of Theorem 2.5 below. Other inequalities known for even ferromagnets include the Griffiths-Kelley-Sherman (GKS I and II) inequalities. They were originally proved for spin-1/2 Ising models $[8,13]$ and have since been shown for all measures in $\mathscr{E}$ and for Hamiltonians more general than (1.2) (see [4; 26, p. 274] and Appendix A of this paper). In contrast, measures in $\mathscr{E}$ for which the GHS inequality fails are easily obtained $[12$, p. 153]: for example,

$$
\varrho_{a}(d x)=a \delta(x)+((1-a) / 2)(\delta(x-1)+\delta(x+1)) \text { for } 2 / 3<a<1 .
$$

The GHS inequality has a number of interesting implications: concavity of the average magnetization $N^{-1} \sum_{i=1}^{N} E\left\{X_{i}\right\}$ as a function of the external field [11]; absence of phase transitions for $h_{1}=\ldots=h_{N}>0$ [23]; monotonicity of correlation length in Ising models [14] and of mass gap in $\varphi^{4}$ field theories [12] as functions of the external field; absence of certain bound states in quantum field theory $[6,7]$. It has also been used to derive critical point exponent inequalities [11, p. 795], absolute bounds on physical coupling constants in quantum field theory [5], and eigenvalue inequalities in quantum mechanics. For the latter, see the remark before Theorem 1.2 below.

Our main result is to give conditions on the measures $\varrho_{i}$ which guarantee (1.4). This is done in terms of a subclass $\mathscr{G}_{-}$of $\mathscr{E}$, defined at the beginning of Section 2.

Theorem 1.1. If $\varrho_{1}, \ldots, \varrho_{N}$ are in $\mathscr{G}_{-}$, then the GHS inequality (1.4) holds.

Essentially, a measure belongs to $\mathscr{G}_{-}$if an infinite string of moment inequalities are satisfied. These inequalities were used in [2] to prove the special case of $(1.4)$ 
where $N=1$. The new element needed to prove (1.4) is certain multivariate correlation inequalities satisfied by measures in $\mathscr{G}_{-}$.

Our next result, Theorem 1.2, gives a large class of measures which belong to $\mathscr{G}_{\text {. }}$. This property of measures of the form (1.7) generalizes Theorem 4 in [2], which admitted entire functions $V(x)$ with the expansion

$$
\begin{aligned}
& V(x)=\sum_{k=1}^{\infty} a_{k} x^{2 k}, a_{k} \geqq 0 \text { for } k=2,3, \ldots, a_{1} \text { real }\left(a_{1}>0\right. \\
& \text { if all other } \left.a_{k} \text { are } 0\right) .
\end{aligned}
$$

As an application of Theorems 1.1 and $1.2 \mathrm{c}$ ), it follows that when $V$ satisfies the hypotheses of Theorem 1.2, the first three eigenvalues $E_{1}<E_{2}<E_{3}$ of the quantum mechanical Schrödinger operator $-d^{2} / d x^{2}+V(x)$ on $L^{2}(\mathbb{R} ; d x)$ satisfy $E_{3}-E_{2} \geqq$ $E_{2}-E_{1}$ [24]. It also follows that the gap $E_{2}(a)-E_{1}(a)$ between the first two eigenvalues $E_{1}(a)$ and $E_{2}(a)$ of the operator $-d^{2} / d x^{2}+V(x)+a x$ on $L^{2}(\mathbb{R} ; d x)$ increases with $a[26$, p. 335].

Theorem 1.2. a) $\mathscr{G}_{\text {_ }}$ contains the Bernoulli measure $b(d x)$.

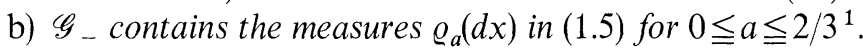

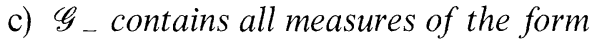

$$
\varrho_{V}(d x)=\left(\int_{\mathbb{R}} \exp (-V(x)) d x\right)^{-1} \exp (-V(x)) d x,
$$

where $V(x)$ is even, continuously differentiable, and unbounded above at infinity, and $V^{\prime}(x)$ is convex on $[0, \infty)$ (' denotes $d / d x$ ).

d) $\mathscr{G}_{-}$contains all absolutely continuous measures $\varrho \in \mathscr{E}$ with support on $[-a, a]$ for some $0<a<\infty$ provided $g(x)=d \varrho / d x$ is continuously differentiable and strictly positive on $(-a, a)$ and $g^{\prime}(x) / g(x)$ is concave on $[0, a)$.

That $b(d x)$ belongs to $\mathscr{G}_{\text {_ }}$ was first noted in [2]; we give another proof.

Combining Theorems 1.1 and 1.2, we obtain a direct proof of the GHS inequality for spin $-1 / 2$ Ising models, and, for example, for the measures

$$
\begin{aligned}
& \varrho(d x)=\text { const } \times \exp \left(-a x^{4}-b x^{2}\right) d x, \\
& \varrho(d x)=\text { const } \times \exp \left(-a \cosh x-b x^{2}\right) d x,
\end{aligned}
$$

where $a>0$ and $b$ is real. Both of these are of physical interest (for $(1.9)$, see $[26, \S 5.6]$ ). Since the measure in (1.8) has been shown to be ferromagnetic, the GHS inequality holds for it [12], but until now a direct proof has been lacking. We point out that ferromagnetic families of random variables satisfy other properties, such as the Lee-Yang theorem, which are distinct from (1.4) [20].

In Section 2 of this paper, we define the class $\mathscr{G}_{-}$and prove the multivariate correlation inequalities needed for the proof of Theorem 1.1. We also derive other properties of this class. In Section 3 we prove Theorem 1.1. Our idea is to use the multivariate $\mathscr{G}_{\text {_ }}$ inequalities to show that the multi-Taylor coefficients in the expansion of the entire function

$$
\left[Z\left(h_{1}, \ldots, h_{N}\right)\right]^{4} \partial^{3} Z\left(h_{1}, \ldots, h_{N}\right) / \partial h_{i} \partial h_{j} \partial h_{k}
$$

1 By an argument of Preston [23], this implies that the tricritical point in Griffiths-type models of $\mathrm{He}^{3}-\mathrm{He}^{4}$ mixtures (see $[1,10]$ ) is not less than $2 / 3$. 
in powers of $h_{l}, l=1, \ldots, N$, are all negative. We also note other correlation inequalities, one of which appears to be new:

$$
\langle i j k l\rangle-\langle i j\rangle\langle k l\rangle-\langle i k\rangle\langle j l\rangle-\langle i l\rangle\langle j k\rangle+2\langle i\rangle\langle j\rangle\langle k\rangle\langle l\rangle \leqq 0,
$$

where $i, j, k, l$ are any (not necessarily distinct) sites in $\Lambda$ and $\langle i j k l\rangle=E\left\{X_{i} X_{j} X_{k} X_{l}\right\}$, etc. In Appendix A, we give simple proofs of the first and second GKS inequalities as well as other correlation inequalities valid for arbitrary measures in $\mathscr{E}$. One of these is new:

$$
\sum_{k=0}^{n}(-1)^{k} \sum\left\langle j_{1} \ldots j_{k}\right\rangle\left\langle j_{k+1} \ldots j_{2 n}\right\rangle \geqq 0, n=1,2, \ldots,
$$

where the inner summation runs over all partitions of a fixed set $\left\{i_{1}, \ldots, i_{2 n}\right\}$ of (not necessarily distinct) sites of $\Lambda$ into two subsets $\left\{j_{1}, \ldots, j_{k}\right\},\left\{j_{k+1}, \ldots, j_{2 n}\right\}$ and the empty expectations equal one. In Appendix B, we modify our methods to prove GKS-type inequalities for systems with

$$
\varrho_{i}(d x)=(1 / 3)(\delta(x-1)+\delta(x)+\delta(x+1)), \quad i=1, \ldots, N,
$$

and with the following Hamiltonians which are different from (1.2):

$$
\begin{aligned}
H\left(x_{1}, \ldots, x_{N}\right)= & -\sum_{1 \leqq i \leqq j \leqq N} J_{i j}\left(a x_{i}^{2}-b x_{i}\right)\left(a x_{j}^{2}-b x_{j}\right)+b \sum_{1 \leqq i \leqq N} h_{i} x_{i} \\
& -\sum_{1 \leqq i \leqq N} \mu_{i} x_{i}^{2}, \\
H\left(x_{1}, \ldots, x_{N}\right)= & -\sum_{1 \leqq i \leqq j \leqq N} J_{i j} x_{i} x_{j}-\sum_{1 \leqq i \leqq j \leqq N} \gamma_{i j}\left(a x_{i}^{2}-b\right)\left(a x_{j}^{2}-b\right) \\
& -\sum_{1 \leqq i \leqq N} h_{i} x_{i},
\end{aligned}
$$

with $J_{i j} \geqq 0, \gamma_{i j} \geqq 0, h_{i} \geqq 0, \mu_{i}$ real, $a$ and $b$ real. Such systems have been used to model ternary mixtures [16] and $\mathrm{He}^{3}-\mathrm{He}^{4}$ mixtures [1].

One contribution of the present work is that it proves the GKS and GHS inequalities by a unified technique. The proof of each employs independent, identically distributed copies of random variables (one for GKS I, two for GKS II, four for GHS) as well as a special orthogonal matrix used to rotate the independent copies. The $4 \times 4$ matrix used for GHS is the direct product with itself of the $2 \times 2$ matrix used for GKS II. While the infinite string of correlation inequalities which enter in the proof of GHS are conditions for a measure's belonging to $\mathscr{G}_{-}$, the analogous correlation inequalities needed for GKS are automatically satisfied by all measures in $\mathscr{E}$.

The idea of using two or four independent copies of random variables has been employed to good advantage by other workers in the field $[4,15,22,27,29]$. No use has yet been found for eight or more independent copies.

As this paper was being prepared, we learned of new work of Sylvester [27], who has independently proved Theorem 1.1 and who has shown that the $\mathscr{G}_{-}$ inequalities imply the correlation inequalities of Lebowitz [15]. Similar results have also been obtained by Schrader and Mehta (unpublished).

Acknowledgment. One of us (R.S.E.) has benefited from conversations with the following people: A. Devinatz, J. Glimm, A. Jaffe, J. Lebowitz, M. Marcus, J. Percus, S. Sawyer, G. Sylvester, and S. Varadhan. Another of us (C.M.N.) would like to thank A. Lenard and S. Sherman for many useful conversations. 


\section{Facts about $\mathscr{G}_{-}$}

Our definition of $\mathscr{G}_{-}$is taken from [2]. After giving it, we indicate an alternate form which simplifies calculations.

Definition 2.1. Given $\varrho \in \mathscr{E}$, let $W^{(\alpha)}, \alpha=1, \ldots, 4$, be four independent copies of a random variable $W$ distributed by $\varrho$. Let $W=\left(W^{(1)}, \ldots, W^{(4)}\right)$ and $\boldsymbol{m}=$ $\left(m^{(1)}, \ldots, m^{(4)}\right)$, where each $m^{(\alpha)}$ is a non-negative integer. We say that $\boldsymbol{m}$ is odd (resp., even) if each $m^{(\alpha)}$ is an odd (resp., even) integer. Take $A$ to be the orthogonal matrix

$$
A=1 / \sqrt{2}\left(\begin{array}{rr}
1 & 1 \\
-1 & 1
\end{array}\right) \otimes 1 / \sqrt{2}\left(\begin{array}{rr}
1 & 1 \\
-1 & 1
\end{array}\right)=1 / 2\left(\begin{array}{rrrr}
1 & 1 & 1 & 1 \\
-1 & 1 & -1 & 1 \\
-1 & -1 & 1 & 1 \\
1 & -1 & -1 & 1
\end{array}\right)
$$

and define

$$
(A \boldsymbol{W})^{(\alpha)}=\sum_{\beta=1}^{4} A_{\alpha, \beta} W^{(\beta)},(A \boldsymbol{W})^{\boldsymbol{m}}=\prod_{\alpha=1}^{4}\left[(A \boldsymbol{W})^{(\alpha)}\right]^{m^{(\alpha)}} .
$$

We define

$$
\mathscr{G}_{-}=\left\{\varrho \in \mathscr{E}: E\left\{(A \boldsymbol{W})^{\boldsymbol{m}}\right\} \leqq 0 \text { for all } \boldsymbol{m} \text { odd }\right\} .
$$

Remark 2.2. Given $\varrho \in \mathscr{E}$ (resp., $\varrho \in \mathscr{G}_{-}$) and $W$ distributed by $\varrho$, we shall write $W \in \mathscr{E}$ (resp., $W \in \mathscr{G}_{-}$). that

Sylvester [28] has noted that the definition of $\mathscr{G}_{-}$is equivalent to requiring

$$
E\left\{(B \boldsymbol{W})^{\boldsymbol{m}}\right\} \geqq 0 \text { for all } \boldsymbol{m},
$$

where $B$ is the matrix

$$
B=1 / 2\left(\begin{array}{rrrr}
1 & 1 & 1 & 1 \\
1 & -1 & 1 & -1 \\
1 & 1 & -1 & -1 \\
-1 & 1 & 1 & -1
\end{array}\right)
$$

This is so because $B$ is obtained from $A$ by multiplying an odd number of rows by -1 and because the expectation $E\left\{(A \boldsymbol{W})^{\boldsymbol{m}}\right\}$ vanishes unless $\boldsymbol{m}$ is either even (in which case the expectation is clearly non-negative) or odd. The latter fact is proved as Part (c) of Theorem 2.5. We define

$$
\mu_{\varrho}(\boldsymbol{m})=\mu_{W}(\boldsymbol{m})=E\left\{(B \boldsymbol{W})^{\boldsymbol{m}}\right\} .
$$

Warning. In [2], $\mu_{\varrho}(\boldsymbol{m})$ denotes $E\left\{(A \boldsymbol{W})^{\boldsymbol{m}}\right\}$.

Our proof of Theorem 1.1, given in Section 3, is based upon multivariate versions of the $\mathscr{G}_{-}$inequalities (2.2). We prove the following result at the end of this section.

Theorem 2.3. Let $\left\{Y_{1}, \ldots, Y_{N}\right\}$ be a set of real-valued random variables with joint distribution (see (1.1))

$$
\left.\tau_{0} \equiv \tau_{h_{1}, \ldots, h_{N}}\right|_{\text {all } h_{l}=0} \text {. }
$$

Let $\left\{Y_{1}^{(\alpha)}, \ldots, Y_{N}^{(\alpha)}\right\}, \alpha=1,2,3,4$, be four independent copies of $\left\{Y_{1}, \ldots, Y_{N}\right\}$ and define

$$
\boldsymbol{Y}_{i}=\left(Y_{i}^{(1)}, \ldots, Y_{i}^{(4)}\right) .
$$


Given multi-indices $\boldsymbol{m}_{1}, \ldots, \boldsymbol{m}_{N}$, we define

$$
\mu\left(\boldsymbol{m}_{1}, \ldots, \boldsymbol{m}_{N}\right)=\mu_{Y_{1} \ldots Y_{N}}\left(\boldsymbol{m}_{1}, \ldots, \boldsymbol{m}_{N}\right)=E\left\{\prod_{i=1}^{N}\left(B \boldsymbol{Y}_{i}\right)^{\boldsymbol{m}_{i}}\right\} .
$$

If $\varrho_{1}, \ldots, \varrho_{N} \in \mathscr{G}_{-}$, then

$$
\mu\left(\boldsymbol{m}_{1}, \ldots, \boldsymbol{m}_{N}\right) \geqq 0 \text { for all } \boldsymbol{m}_{1}, \ldots, \boldsymbol{m}_{N} .
$$

We state additional facts about $\mathscr{G}_{-}$(proved at the end of this section) after the next definition.

Definition 2.4. Let $Y_{1}, \ldots, Y_{N}$ be random variables as in Theorem 2.3. If $Y=\sum_{i=1}^{N} \lambda_{i} Y_{i}$ for some $\lambda_{i} \geqq 0$, then $Y$ is a ferromagnetic union of the $\left\{Y_{i}\right\}$.

Theorem 2.5. (a) Given $Y_{1}, \ldots, Y_{N}$ as in Theorem 2.3 with each $\varrho_{i} \in \mathscr{G}_{-}$, then any ferromagnetic union of the $\left\{Y_{i}\right\}$ belongs to $\mathscr{G}_{-}$. (b) If $W_{n}, n=1,2, \ldots$, are random variables in $\mathscr{G}_{-}$such that $W=w-\lim _{n \rightarrow \infty} W_{n}$ exists (i.e., the probability distribution of the $W_{n}$ converge weakly to the probability distribution of $W$ ) and $\sup _{n} E\left\{W_{n}^{2}\right\} \leqq$ $K<\infty$, then $W \in \mathscr{G}_{-}$. (c) Given $\varrho \in \mathscr{E}$, then $\mu_{\varrho}(\boldsymbol{m})>0$ if $\boldsymbol{m}$ is even and $\mu_{\varrho}(\boldsymbol{m})=0$ if $\boldsymbol{m}$ is neither even nor odd. (d) The numbers $\mu_{\varrho}(\boldsymbol{m})$ vanish for all $\boldsymbol{m}$ odd if and only if either $\varrho$ is an even Gaussian measure or $\varrho=\delta(x)$.

In $[18, \S 4]$ the set $\mathscr{F}_{0}$ of mean zero ferromagnetic random variables is defined. We shall say that a measure $\varrho \in \mathscr{E}$ is mean zero ferromagnetic if a random variable distributed by $\varrho$ belongs to $\mathscr{F}_{0}$. Using the fact that the Bernoulli measure belongs to $\mathscr{G}_{\text {_ }}$ [see Theorem 1.2(a)], we have by Theorem 2.5(a) and (b) that $\mathscr{F}_{0}$ is a subset of $\mathscr{G}_{\text {. }}$. As examples of measures in $\mathscr{F}_{0}$, we have the measure in (1.8), the measures $\varrho_{a}(d x)$ in (1.5) for $0 \leqq a \leqq \frac{1}{2}$ (as can be shown by direct construction), the measures [9]

$$
b_{c, k}(d x)=(k+1)^{-1} \sum_{j=0}^{k} \delta(c(1-2 j / k)), \quad k=1,2, \ldots, \quad 0<c<\infty,
$$

and normalized Lebesgue measure on $[-c, c]$, any $0<c<\infty$ [weak limit of $b_{c, k}(d x)$ as $\left.k \rightarrow \infty\right]$.

We claim that $\mathscr{F}_{0}$ is actually a proper subset of $\mathscr{G}_{-}$. Indeed, the measures $\varrho_{a}(d x)$ for $0 \leqq a \leqq 2 / 3$ belong to $\mathscr{G}_{-}$[see Theorem $\left.1.2(\mathrm{~b})\right]$ while for $1 / 2<a<1$ these measures are not mean zero ferromagnetic. To see the latter fact, we note that any mean zero ferromagnetic measure satisfies the Lee-Yang condition [18, Remark 5], which is that

the entire function $h \rightarrow \int e^{h x} \varrho(d x)$ has pure imaginary zeroes in $h$.

It is easy to ckeck that (2.8) is not true for $\varrho_{a}(d x), \frac{1}{2}<a<1$.

We end this discussion with a conjecture concerning the $\mathscr{G}_{-}$inequalities and another set of inequalities, called the Khintchine inequalities. The latter have single-site and multivariate forms. In their single-site form, the Khintchine inequalities state that the moments of $\varrho \in \mathscr{E}$ are bounded by the corresponding moments of a mean zero Gaussian measure with the same variance as $\varrho$; they are known to hold for any measure in $\mathscr{E}$ which satisfies (2.8). In their multivariate form they are known to hold for any mean zero ferromagnetic family of measures. 
See [19, Theorem 5] and [18, Theorem 3] for complete statements of these inequalities and proofs of the above facts and [25] for an application of the multivariate Khintchine inequalities in field theory.

Based on calculations with the first four $\mathscr{G}_{\text {_ }}$ inequalities, we make the following conjecture.

Conjecture. Given $Y_{1}, \ldots, Y_{N}$ as in Theorem 2.3 with each $\varrho_{i} \in \mathscr{G}_{-}$, then the $Y_{i}^{\prime} \mathrm{s}$ satisfy the multivariate Khintchine inequalities. In particular, any $\varrho \in \mathscr{G}_{-}$satisfies the single-site Khintchine inequalities.

As further evidence, we consider the measures $\varrho_{a}(d x)$ in (1.5). By Theorem 1.2(b) these measures belong to $\mathscr{G}_{-}$for $0 \leqq a \leqq 2 / 3$. One can verify that the $\varrho_{a}(d x)$ satisfy the single-site Khintchine inequalities for exactly the same values of $a$. We also note that it is precisely for Gaussian measures or the trivial measure that all the single-site $\mathscr{G}_{-}$inequalities and all the single-site Khintchine inequalities are equalities.

Proof of Theorem 2.3. For each $i=1, \ldots, N$, let $W_{i}^{(\alpha)}, a=1, \ldots, 4$, be four independent copies of a random variable distributed by $\varrho_{i}$. We assume that $W_{i}^{(\beta)}$ and $W_{j}^{(\gamma)}$ are independent unless $i=j$ and $\beta=\gamma$. We set

$$
\boldsymbol{W}_{i}=\left(W_{i}^{(1)}, \ldots, W_{i}^{(4)}\right) \text {. }
$$

By the definition of $\boldsymbol{Y}_{i}$ and the orthogonality of $B$, we have

$$
\begin{aligned}
Z_{0}^{4} E\left\{\prod_{i=1}^{N}\left(B \boldsymbol{Y}_{i}\right)^{\boldsymbol{m}_{i}}\right\} & =E\left\{\prod_{i=1}^{N}\left(B \boldsymbol{W}_{i}\right)^{\boldsymbol{m}_{i}} \exp \left(\sum J_{i j} \boldsymbol{W}_{i} \cdot \boldsymbol{W}_{j}\right)\right\} \\
& =E\left\{\prod_{i=1}^{N}\left(B \boldsymbol{W}_{i}\right)^{\boldsymbol{m}_{i}} \exp \left(\sum J_{i j}\left(B \boldsymbol{W}_{i}\right) \cdot\left(B \boldsymbol{W}_{j}\right)\right)\right\}
\end{aligned}
$$

where $Z_{0}=\left.Z\left(h_{1}, \ldots, h_{N}\right)\right|_{\text {all } h_{i}=0}$ and . denotes the standard $\mathbb{R}^{4}$ inner product. Expanding the exponential and using the positivity of the $J_{i j}$, we see that it suffices to prove that

$$
E\left\{\prod_{i=1}^{N}\left(B \boldsymbol{W}_{i}\right)^{\boldsymbol{n}_{2}}\right\} \geqq 0 \text { for arbitrary indices } \boldsymbol{n}_{i} .
$$

But (2.10) follows from the independence of the $\boldsymbol{W}_{i}$ and from the hypothesis that each $\varrho_{i}$ is in $\mathscr{G}_{-}$. This completes the proof.

Proof of Theorem 2.5. For Part (a) it follows from Theorem 2.3 that for any $\boldsymbol{m}$ and $\lambda_{i} \geqq 0$

$$
E\left\{\left[B\left(\sum_{i=1}^{N} \lambda_{i} Y_{i}\right)\right]^{m}\right\} \geqq 0 .
$$

For Part (b), since each $W_{n} \in \mathscr{G}_{-}$, we have by Theorem 1.1 and [19, Proposition 1] that for all real $h$

$$
E\left\{\exp \left(h W_{n}\right)\right\} \leqq \exp \left(h^{2} E\left\{W_{n}^{2}\right\} / 2\right) \leqq \exp \left(h^{2} K / 2\right), \quad n=1,2, \ldots
$$

Hence (as in [19, Theorem 5])

$$
\lim _{n \rightarrow \infty} E\left\{W_{n}^{2 m}\right\}=E\left\{W^{2 m}\right\}, \quad m=1,2, \ldots
$$

Since each $\mu_{W_{n}}(\boldsymbol{m}) \geqq 0$ for all $\boldsymbol{m}$, this carries over to $\mu_{W}(\boldsymbol{m}) \geqq 0$ for all $\boldsymbol{m}$. Hence $W \in \mathscr{G}_{-}$. 
The first half of (c) is obvious. For the second half, we use the fact that the random variables $W^{(1)}, \ldots, W^{(4)}$ in the definition of $\mathscr{G}_{\text {- }}$ are symmetric and identically distributed to prove that

$$
\begin{aligned}
\mu_{\varrho}(\boldsymbol{m})= & \left(\mu_{\varrho}(\boldsymbol{m}) / 4\right)\left[(-1)^{m^{(2)}+m^{(3)}}+(-1)^{m^{(2)}+m^{(4)}}+(-1)^{m^{(3)}+m^{(4)}}\right. \\
& \left.+(-1)^{m^{(1)}+m^{(2)}+m^{(3)}+m^{(4)}}\right] .
\end{aligned}
$$

From this it follows that $\mu_{\varrho}(\boldsymbol{m})=0$ if $\boldsymbol{m}$ is neither even nor odd.

The sufficiency of the condition in (d) is checked by explicit calculation. For the necessity let us assume only that

$$
\mu_{\varrho}\left(\boldsymbol{m}_{j}\right)=0, \text { all } j \text { odd, where } \boldsymbol{m}_{j}=(j, 1,1,1) \text {. }
$$

For $N=1,(3.12)$ below (also see [2, Eq. (8)]) becomes

$$
d^{3} / d h^{3} \ln \int \exp (h x) \varrho(d x)=-2[Z(h)]^{-4} \sum_{j \text { odd }} j !^{-1}(2 h)^{j} \mu_{\varrho}\left(\boldsymbol{m}_{j}\right),
$$

and so by (2.12)

$$
d^{3} / d h^{3} \ln \int \exp (h x) \varrho(d x)=0 .
$$

Thus $\int \exp (h x) \varrho(d x)=a \exp \left(b h^{2}\right), a$ and $b$ real, and so $\varrho=\delta(x)$ if $b=0$, Gaussian otherwise. This completes the proof of the theorem.

\section{Proof of Theorem 1.1}

Given (not necessarily distinct) sites $i_{1}, \ldots, i_{n} \in \Lambda, n=1,2, \ldots$, we define the Ursell functions

$$
U_{n}\left(i_{1}, \ldots, i_{n}\right)=\partial^{n} / \partial h_{i_{1}} \ldots \partial h_{i_{n}} \ln Z\left(h_{1}, \ldots, h_{N}\right)
$$

and set

$$
U_{n}^{(0)}\left(i_{1}, \ldots, i_{n}\right)=\left.U_{n}\left(i_{1}, \ldots, i_{n}\right)\right|_{\text {all } h_{i}=0} .
$$

We write $Z$ for $Z\left(h_{1}, \ldots, h_{N}\right)$ and let

$$
\left\langle j_{1} \ldots j_{m}\right\rangle=E\left\{X_{j_{1}} \ldots X_{j_{m}}\right\},\left\langle j_{1} \ldots j_{m}\right\rangle_{0}=\left.E\left\{X_{j_{1}} \ldots X_{j_{m}}\right\}\right|_{\text {all } h_{i}=0} .
$$

Given $i, j, k \in \Lambda$, we have

$$
U_{3}(i, j, k)=\langle i j k\rangle-\langle i\rangle\langle j k\rangle-\langle j\rangle\langle i k\rangle-\langle k\rangle\langle i j\rangle+2\langle i\rangle\langle j\rangle\langle k\rangle .
$$

We define vectors

$$
\boldsymbol{h}_{l}=\left(h_{l}^{(1)}, \ldots, h_{l}^{(4)}\right), l=1, \ldots, N,
$$

and consider random vectors $\boldsymbol{Y}_{l}$ as in (2.5). We rewrite $U_{3}(i, j, k)$ as

$$
U_{3}(i, j, k)=\left.\left(Z_{0} / Z\right)^{4} E\left\{\mathscr{D} \exp \left(\sum_{l=1}^{N} \boldsymbol{h}_{l} \cdot \boldsymbol{Y}_{l}\right)\right\}\right|_{h_{l}^{(\alpha)}=h_{l}, \alpha=1, \ldots, 4},
$$

where $\mathscr{D}$ is the differential operator

$$
\begin{aligned}
\mathscr{D}= & \partial^{3} / \partial h_{i}^{(1)} \partial h_{j}^{(1)} \partial h_{k}^{(1)}-\partial^{3} / \partial h_{i}^{(2)} \partial h_{j}^{(1)} \partial h_{k}^{(1)}-\partial^{3} / \partial h_{i}^{(1)} \partial h_{j}^{(2)} \partial h_{k}^{(1)} \\
& -\partial^{3} / \partial h_{i}^{(1)} \partial h_{j}^{(1)} \partial h_{k}^{(2)}+2 \partial^{3} / \partial h_{i}^{(1)} \partial h_{j}^{(2)} \partial h_{k}^{(3)} .
\end{aligned}
$$


Define

$$
\boldsymbol{s}_{l}=\left(s_{l}^{(1)}, \ldots, s_{l}^{(4)}\right)=\boldsymbol{h}_{l} B^{t}, l=1, \ldots, N .
$$

As in [3, Appendix] (where the matrix $A$ was used instead of $B$ ), we find that

$$
U_{3}(i, j, k)=-2\left(Z_{0} / Z\right)^{4} \tilde{E}\left\{\mathscr{D}^{\prime} \exp \left(\sum_{l=1}^{N} s_{l} \cdot\left(B \boldsymbol{Y}_{l}\right)\right)\right\},
$$

where

$$
\mathscr{D}^{\prime}=\partial^{3} / \partial s_{i}^{(2)} \partial s_{j}^{(3)} \partial s_{k}^{(4)}
$$

and $\tilde{E}$ denotes expectation followed by evaluation at

$$
s_{l}^{(1)}=2 h_{l}, s_{l}^{(2)}=s_{l}^{(3)}=s_{l}^{(4)}=0, l=1, \ldots, N .
$$

The latter corresponds to the $h_{l}^{(\alpha)}$ evaluation in (3.6) since

$$
\left(h_{l}, h_{l}, h_{l}, h_{l}\right) B^{t}=\left(2 h_{l}, 0,0,0\right) .
$$

Expanding the exponential in (3.8), we see that

$$
\begin{aligned}
& U_{3}(i, j, k) \\
& =-2\left(Z_{0} / Z\right)^{4}\left[\mathscr{D}^{\prime} \sum\left(\left(\boldsymbol{s}_{1}\right)^{\boldsymbol{m}_{1}} / \boldsymbol{m}_{1} !\right) \ldots\left(\left(\boldsymbol{s}_{N}\right)^{\boldsymbol{m}_{N}} / \boldsymbol{m}_{N} !\right) \mu_{Y_{1} \ldots Y_{N}}\left(\boldsymbol{m}_{1}, \ldots, \boldsymbol{m}_{N}\right)\right],
\end{aligned}
$$

where the sum extends over all multi-indices $\boldsymbol{m}_{1}, \ldots, \boldsymbol{m}_{N}$ and

$$
\boldsymbol{m}_{l} !=\prod_{\alpha=1}^{4}\left(m_{l}^{(\alpha)}\right) ! .
$$

Carrying out the evaluation given by (3.10), we find that

$$
\begin{aligned}
U_{3}(i, j, k)= & \left.-2\left(Z_{0} / Z\right)^{4} \sum_{\substack{1 \leqq n_{l}<\infty \\
l=1, \ldots, N}}\left(\left(2 h_{1}\right)^{n_{1}} / n_{1} !\right) \ldots\left(2 h_{N}\right)^{n_{N}} / n_{N} !\right) \\
& \cdot \mu_{Y_{1} \ldots Y_{N}}\left(\boldsymbol{n}_{1}, \ldots, \boldsymbol{n}_{N}\right),
\end{aligned}
$$

where

$$
\boldsymbol{n}_{i}=\left(n_{i}, 1,0,0\right), \boldsymbol{n}_{j}=\left(n_{j}, 0,1,0\right), \boldsymbol{n}_{k}=\left(n_{k}, 0,0,1\right),
$$

and $\boldsymbol{n}_{l}=\left(n_{l}, 0,0,0\right)$ for $l \neq i, j, k$.

By Theorem 2.3, the proof is complete.

Remark 3.1. Sylvester [28] has proved this in slightly different form. He sets

$$
\boldsymbol{h}_{i}^{\prime}=\left(h_{i}, h_{i}, h_{i}, h_{i}\right)
$$

and writes

$$
\begin{aligned}
U_{3}(i, j, k) & =-\left(Z_{0} / Z\right)^{4} E\left\{\left(B \boldsymbol{Y}_{i}\right)^{(2)}\left(B \boldsymbol{Y}_{j}\right)^{(3)}\left(B \boldsymbol{Y}_{k}\right)^{(4)} \exp \left(\sum_{l=1}^{N} \boldsymbol{h}_{l}^{\prime} \cdot \boldsymbol{Y}_{l}\right)\right\} \\
& =-\left(Z_{0} / Z\right)^{4} E\left\{\left(B \boldsymbol{Y}_{i}\right)^{(2)}\left(B \boldsymbol{Y}_{j}\right)^{(3)}\left(B \boldsymbol{Y}_{k}\right)^{(4)} \exp \left(\sum_{l=1}^{N} h_{l}\left(B \boldsymbol{Y}_{l}\right)^{(1)}\right)\right\},
\end{aligned}
$$

from which the GHS inequality follows by expanding the exponential and using Theorem 2.3.

Remark 3.2. Other inequalities follow from (3.12). Under the same hypotheses as in Theorem 1.1, we have

$$
\partial^{n} /\left.\partial h_{i_{1}} \ldots \partial h_{i_{n}}\left\{Z^{4}\left(h_{1}, \ldots, h_{N}\right) U_{3}(i, j, k)\right\}\right|_{h_{1}=\ldots=h_{N}=0} \leqq 0
$$


for all $i, j, k, i_{1}, \ldots, i_{n} \in \Lambda, n=0,1,2, \ldots$. For $n=1$, we obtain

$$
U_{4}^{(0)}\left(i_{1}, i_{2}, i_{3}, i_{4}\right) \leqq 0
$$

and for $n=3$

$$
U_{6}^{(0)}\left(i_{1}, i_{2}, i_{3}, i_{4}, i_{5}, i_{6}\right) \leqq-(4 / 5) \sum_{1}\left\langle i_{1}^{\prime} i_{2}^{\prime}\right\rangle_{0} U_{4}^{(0)}\left(i_{3}^{\prime}, i_{4}^{\prime}, i_{5}^{\prime}, i_{6}^{\prime}\right),
$$

where $\sum_{1}$ extends over the 15 different partitions of $\left\{i_{1}, \ldots, i_{6}\right\}$ into subsets $\left\{i_{1}^{\prime}, i_{2}^{\prime}\right\}$ and $\left\{i_{3}^{\prime}, \ldots, i_{6}^{\prime}\right\}$. Using [18, Eq. (3.24)], which is valid for a mean zero ferromagnetic family of random variables $[18, \S 4]$, we obtain from $(3.15)$ for the latter type system

$$
U_{6}^{(0)}\left(i_{1}, \ldots, i_{6}\right) \leqq(8 / 5) \sum_{2}\left\langle i_{1}^{\prime} i_{2}^{\prime}\right\rangle_{0}\left\langle i_{3}^{\prime} i_{4}^{\prime}\right\rangle_{0}\left\langle i_{5}^{\prime} i_{6}^{\prime}\right\rangle_{0},
$$

where $\sum_{2}$ extends over the 15 different partitions of $\left\{i_{1}, \ldots, i_{6}\right\}$ into subsets $\left\{i_{2}^{\prime}, i_{2}^{\prime}\right\}$, $\left\{i_{3}^{\prime}, i_{4}^{\prime}\right\},\left\{i_{5}^{\prime}, i_{6}^{\prime}\right\}$.

As explained in [3, Theorem 1], further inequalities can be obtained from different choices of the derivative operator $\mathscr{D}^{\prime}$ in (3.8). For example, (1.10) follows if we let

$$
\mathscr{D}^{\prime}=\partial^{4} / \partial s_{i}^{(1)} \partial s_{j}^{(2)} \partial s_{k}^{(3)} \partial s_{l}^{(4)} \text {. }
$$

\section{Proof of Theorem 1.2}

By the discussion following (2.3), in order to show that a given $\varrho \in \mathscr{E}$ belongs to $\mathscr{G}_{-}$, we need only show

$$
E\left\{(B \boldsymbol{W})^{\boldsymbol{m}}\right\} \geqq 0 \text { for all } \boldsymbol{m} \text { odd . }
$$

We first obtain an alternative expression for the expectation in (4.1) and then derive a sufficient condition for (4.1) to hold. We define

$$
\mathbb{R}_{+}^{4}=\left\{\boldsymbol{w}=\left(w^{(1)}, w^{(2)}, w^{(3)}, w^{(4)}\right) \in \mathbb{R}^{4}: \text { each } w^{(\alpha)}>0\right\}
$$

and denote by $\left(\mathbb{R}_{+}^{4}\right)^{\text {cl }}$ the closure of $\mathbb{R}_{+}^{4} \cdot \mathbb{R}_{+}^{3}$ and $\left(\mathbb{R}_{+}^{3}\right)^{\mathrm{cl}}$ are defined analogously. To ease the notation in this section, we denote $\boldsymbol{w}$ by $w$. $\mathbb{R}^{4}$ by

Given $\varrho \in \mathscr{E}$ and $T$ a $4 \times 4$ invertible matrix, we define the measure $\varrho_{T}$ on

$$
\varrho_{T}(F)=\varrho\left(T^{-1} F\right), \quad F \text { a Borel set in } \mathbb{R}^{4},
$$

where $\varrho(d w)$ is the product measure $\prod_{\alpha=1}^{4} \varrho\left(d w^{(\alpha)}\right)$.

Proposition 4.1. Given $\varrho \in \mathscr{E}$, define the signed measure $\varrho(\varrho)$ on $\mathbb{R}^{4}$ by

$$
\varrho_{\varrho}(d w)=\varrho_{B}(d w)-\varrho_{A}(d w),
$$

where $B$ and $A$ are the orthogonal matrices in (2.3) and (2.1) respectively. Then

$$
\left.E\{B \boldsymbol{W})^{\boldsymbol{m}}\right\}=8 \int_{\mathbb{R}^{4}} w^{\boldsymbol{m}} \underline{\varrho}(d w), \text { for } \boldsymbol{m} \text { odd. }
$$

Hence a sufficient condition for $\varrho$ to belong to $\mathscr{G}_{-}$is that $\varrho$ be a positive measure on $\mathbb{R}_{+}^{4}$. 
Remark 4.2. For future reference we write out $\varrho_{B}$ and $\varrho_{A}$ in case $\varrho$ is absolutely continuous with density $g$ :

$$
\begin{aligned}
\varrho_{B}(d w)= & g\left(\left(w^{(1)}+w^{(2)}+w^{(3)}-w^{(4)}\right) / 2\right) g\left(\left(w^{(1)}-w^{(2)}+w^{(3)}+w^{(4)}\right) / 2\right) \\
& \cdot g\left(\left(w^{(1)}+w^{(2)}-w^{(3)}+w^{(4)}\right) / 2\right) g\left(\left(w^{(1)}-w^{(2)}-w^{(3)}-w^{(4)}\right) / 2\right) \\
& \cdot d w^{(1)} d w^{(2)} d w^{(3)} d w^{(4)}, \\
\varrho_{A}(d w)= & g\left(\left(w^{(1)}-w^{(2)}-w^{(3)}+w^{(4)}\right) / 2\right) g\left(\left(w^{(1)}+w^{(2)}-w^{(3)}-w^{(4)}\right) / 2\right) \\
& \cdot g\left(\left(w^{(1)}-w^{(2)}+w^{(3)}-w^{(4)}\right) / 2\right) g\left(\left(w^{(1)}+w^{(2)}+w^{(3)}+w^{(4)}\right) / 2\right) \\
& \cdot d w^{(1)} d w^{(2)} d w^{(3)} d w^{(4)} .
\end{aligned}
$$

Proof. Given numbers $\sigma^{(\alpha)}= \pm 1, \alpha=1, \ldots, 4$, we denote the diagonal matrix $\left(\sigma^{(\alpha)} \delta_{\alpha \beta}\right), \alpha, \beta=1, \ldots, 4$, by $I(\sigma)$. We have

$$
\begin{aligned}
E\left\{(B \boldsymbol{W})^{\boldsymbol{m}}\right\} & =\int_{\mathbb{R}^{4}} \sum_{\alpha=1}^{4}\left[(B w)^{(\alpha)}\right]^{m^{(\alpha)}} \varrho_{\sim}(d w) \\
& =\int_{\mathbb{R}^{4}} \sum_{\alpha=1}^{4}\left(w^{(\alpha)}\right)^{m^{(\alpha)}} \varrho_{B}(d w) \\
& =\sum_{\sigma^{(1)}, \ldots, \sigma^{(4)}= \pm 1} \int_{\mathbb{R}^{4}} \prod_{\alpha=1}^{4}\left(\sigma^{(\alpha)} w^{(\alpha)}\right)^{m^{(\alpha)}} \varrho_{I(\sigma) B}(d w) \\
& =\int_{\mathbb{R}^{4}} \prod_{\alpha=1}^{4}\left(w^{(\alpha)}\right)^{m^{(\alpha)}}\left[\sum_{\sigma^{(1)}, \ldots, \sigma^{(4)}= \pm 1} \prod_{\alpha=1}^{4}\left(\sigma^{(\alpha)}\right)^{m^{(\alpha)}} \varrho_{I^{(\sigma) B}}(d w)\right] .
\end{aligned}
$$

We now use the fact, which follows from the evenness of $\varrho$, that

$$
\varrho_{I(\sigma) B}(d w)=\left\{\begin{array}{lll}
\varrho_{B}(d w), & \text { if } \prod_{\alpha=1}^{4} \sigma^{(\alpha)}=+1, \\
\varrho_{A}(d w), & \text { if } \prod_{\alpha=1}^{4} \sigma^{(\alpha)}=-1 .
\end{array}\right.
$$

Formula (4.3) follows by the oddness of $\boldsymbol{m}$. This completes the proof.

We next turn to the proof of Theorem 1.2. We shall use the sufficient condition in Proposition 4.1 to prove Parts (a), (c), and (d). To prove Part (b), we will use formula (4.3). Our calculations will indicate that the sufficient condition in the proposition is not necessary.

For Part (a), we have that ${\underset{\sim}{A}}_{A}$ vanishes on $\mathbb{R}_{+}^{4}$ [although it does have support on the boundary of $\mathbb{R}_{+}^{4}$ at the points $(2,0,0,0),(0,2,0,0),(0,0,2,0)$, and $\left.(0,0,0,2)\right]$, and so $\bar{b}$ is a positive measure on $\mathbb{R}_{+}^{4}$.

For Part (b), we use the following easily verified facts: the only points in the support of $\varrho_{a}$ which are mapped to $\mathbb{R}_{+}^{4}$ by $B$ are

$$
\left(\begin{array}{r}
1 \\
1 \\
1 \\
-1
\end{array}\right),\left(\begin{array}{l}
1 \\
1 \\
1 \\
0
\end{array}\right),\left(\begin{array}{r}
1 \\
0 \\
1 \\
-1
\end{array}\right),\left(\begin{array}{r}
1 \\
1 \\
0 \\
-1
\end{array}\right), \quad \text { and } \quad\left(\begin{array}{r}
0 \\
1 \\
1 \\
-1
\end{array}\right)
$$

their images under $B$ are

$$
\left(\begin{array}{l}
1 \\
1 \\
1 \\
1
\end{array}\right), \frac{1}{2}\left(\begin{array}{l}
3 \\
1 \\
1 \\
1
\end{array}\right), \frac{1}{2}\left(\begin{array}{l}
1 \\
3 \\
1 \\
1
\end{array}\right), \frac{1}{2}\left(\begin{array}{l}
1 \\
1 \\
3 \\
1
\end{array}\right), \quad \text { and } \quad \frac{1}{2}\left(\begin{array}{l}
1 \\
1 \\
1 \\
3
\end{array}\right)
$$


respectively; the only point in the support of $\varrho_{a}$ which is mapped to $\mathbb{R}_{+}^{4}$ by $A$ is $\left(\begin{array}{l}0 \\ 0 \\ 0 \\ 1\end{array}\right)$, and $A\left(\begin{array}{l}0 \\ 0 \\ 0 \\ 1\end{array}\right)=\frac{1}{2}\left(\begin{array}{l}1 \\ 1 \\ 1 \\ 1\end{array}\right)$. Hence by (4.3) we have for $\boldsymbol{m}$ odd

$$
E\left\{(B \boldsymbol{W})^{\boldsymbol{m}}\right\}=8 \cdot 2^{-M}\left[((1-a) / 2)^{4} 2^{M}+((1-a) / 2)^{3} a\left(\sum_{\alpha=1}^{4} 3^{m^{(\alpha)}}\right)-a^{3}(1-a) / 2\right],
$$

where $M=\sum_{\alpha=1}^{4} m^{(\alpha)}$. We see that $E\left\{(B W)^{m}\right\} / 8 \cdot 2^{-M}$ is increasing in the $m^{(\alpha)}$ s, and hence (4.1) will follow once we prove

$$
E\left\{(B W)^{(1,1,1,1)}\right\} \geqq 0 \text {. }
$$

But this equals $(1-a)(2-3 a) / 4$, which is non-negative for $0 \leqq a \leqq 2 / 3$. This completes the proof of Part (b). It is clear that the sufficient condition in Proposition 4.1 is not necessary since for $a>0\left(\varrho_{a}\right)_{A}$ has support at the point $\frac{1}{2}\left(\begin{array}{l}1 \\ 1 \\ 1 \\ 1\end{array}\right)$, where $\left(\varrho_{a}\right)_{B}$
has no support.

For Parts (c) and (d), it suffices to prove (c) only. Indeed, given $g(x)$ as in (d), we can find equibounded $C^{1}$ functions $g_{\varepsilon}(x), \varepsilon>0$, such that $\varrho_{\varepsilon}(d x) \equiv g_{\varepsilon}(x) d x /$ $\int g_{\varepsilon}(x) d x \in \mathscr{E}, g_{\varepsilon}>0$ on $\mathbb{R}, g_{\varepsilon}^{\prime} / g_{\varepsilon}$ concave on $(0, \infty), \lim _{\varepsilon \downarrow 0} g_{\varepsilon}(x)=g(x)$ for all $x$, and $\sup _{\varepsilon>0} \int x^{2} g_{\varepsilon}(x) d x<\infty$. By (c) we then have that each $\varrho_{\varepsilon} \in \mathscr{G}_{-}$, and so we conclude

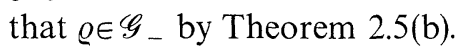

Concerning (c), the hypotheses on $V$ imply that as $x \rightarrow \infty V(x) \geqq \delta x^{2}$ for some $\delta>0$, and so $\varrho_{V} \in \mathscr{E}$. By Proposition 4.1 and formula (4.4), it suffices to prove that

$$
\begin{aligned}
& V\left(w^{(1)}-w^{(2)}-w^{(3)}+w^{(4)}\right)+V\left(w^{(1)}+w^{(2)}-w^{(3)}-w^{(4)}\right) \\
& \quad+V\left(w^{(1)}-w^{(2)}+w^{(3)}-w^{(4)}\right)+V\left(w^{(1)}+w^{(2)}+w^{(3)}+w^{(4)}\right) \\
& \quad-V\left(w^{(1)}+w^{(2)}+w^{(3)}-w^{(4)}\right)+V\left(w^{(1)}+w^{(2)}-w^{(3)}+w^{(4)}\right) \\
& \quad-V\left(w^{(1)}+w^{(2)}-w^{(3)}+w^{(4)}\right)-V\left(w^{(1)}-w^{(2)}-w^{(3)}-w^{(4)}\right) \geqq 0
\end{aligned}
$$

on $\left(\mathbb{R}_{+}^{4}\right)^{\mathrm{cl}}$.

The point is that the expression in (4.8) is an eight point difference approximation to the third derivative of $V$. Part (c) is then a consequence of the following lemma.

Lemma 4.3. Suppose that $V(x)$ is an even $C^{1}$ function on $\mathbb{R}$. Then $V^{\prime}(x)$ is convex on $[0, \infty)$ if and only if $V$ satisfies (4.8).

Proof. We show that (4.8) is equivalent to

$$
\begin{aligned}
& {\left[V^{\prime}\left(w^{(1)}+w^{(2)}+w^{(3)}\right)-V^{\prime}\left(w^{(1)}+w^{(2)}-w^{(3)}\right)\right]} \\
& \quad-\left[V^{\prime}\left(w^{(1)}-w^{(2)}+w^{(3)}\right)-V^{\prime}\left(w^{(1)}-w^{(2)}-w^{(3)}\right)\right] \geqq 0
\end{aligned}
$$

on $\left(\mathbb{R}_{+}^{3}\right)^{\mathrm{cl}}$,

then that (4.9) is equivalent to the convexity. For the first part, we rewrite (4.8) as

$$
\begin{aligned}
& w^{(4)} \int_{-1}^{1}\left\{\left[V^{\prime}\left(w^{(1)}+r w^{(4)}+w^{(2)}+w^{(3)}\right)-V^{\prime}\left(w^{(1)}+r w^{(4)}+w^{(2)}-w^{(3)}\right)\right]\right. \\
& \left.-\left[V^{\prime}\left(w^{(1)}+r w^{(4)}-w^{(2)}+w^{(3)}\right)-V^{\prime}\left(w^{(1)}+r w^{(4)}-w^{(2)}-w^{(3)}\right)\right]\right\} d r \geqq 0
\end{aligned}
$$

on $\left(\mathbb{R}_{+}^{4}\right)^{\mathrm{cl}}$. 
By taking $w^{(4)}=\varepsilon>0$, dividing both sides of (4.10) by $\varepsilon$, and letting $\varepsilon \rightarrow 0$, we see that (4.8) implies (4.9). Conversely, since we may assume by symmetry that $w^{(1)} \geqq w^{(4)}$, we see that if (4.9) holds, then the integrand in (4.10) is non-negative for all $-1 \leqq r \leqq 1$, and so (4.8) follows. Hence (4.8) and (4.9) are equivalent. Now (4.9) clearly implies that

$$
\begin{aligned}
& V^{\prime}(y+k)-V^{\prime}(y) \geqq V^{\prime}(x+k)-V^{\prime}(x), \\
& \text { for all } 0 \leqq x \leqq y \quad \text { and } k \geqq 0,
\end{aligned}
$$

and (4.11) is equivalent to convexity on $[0, \infty)$ for continuous $V^{\prime}$. On the other hand, assuming the oddness and the convexity on $[0, \infty)$ of $V^{\prime}$, we have (4.11) together with (we claim)

$$
V^{\prime}(y+k)-V^{\prime}(y) \geqq V^{\prime}(x)-V^{\prime}(x-k), \text { for all } 0 \leqq x \leqq y \text { and } k \geqq 0 .
$$

Let us accept (4.12) for a moment. We now prove that (4.11) and (4.12) imply (4.9). By symmetry, we may assume that in (4.9) $w^{(1)} \geqq w^{(2)} \geqq w^{(3)} \geqq 0$. When $w^{(1)} \geqq w^{(2)}+$ $w^{(3)}$, then (4.9) follows from (4.11) (take $x=w^{(1)}-w^{(2)}-w^{(3)}, y=w^{(1)}+w^{(\overline{2})}-w^{(3)}$, and $\left.k=2 w^{(3)}\right)$. When $w^{(1)}<w^{(2)}+w^{(3)}$, then (4.9) follows from (4.12) (take $x=w^{(1)}-$ $w^{(2)}+w^{(3)}, y=w^{(1)}+w^{(2)}-w^{(3)}$, and $\left.k=2 w^{(3)}\right)$. We are finished except for (4.12), for which we need only consider the case $x-k<0$. Then

$$
\begin{aligned}
& V^{\prime}(x)-V^{\prime}(x-k)=V^{\prime}(x)+V^{\prime}(k-x)=V^{\prime}(x)+\left(V^{\prime}(k-x)-V^{\prime}(0)\right) \\
& \leqq V^{\prime}(x)+\left(V^{\prime}(x+k-x)-V^{\prime}(x)\right)=V^{\prime}(k)=V^{\prime}(k)-V^{\prime}(0) \leqq V^{\prime}(y+k)-V^{\prime}(y),
\end{aligned}
$$

where we have used (4.11) in the third step and in the last step. This completes the proof.

Remark 4.4. If $V(x)=\sum_{k=0}^{\infty} a_{k} x^{2 k}$ is entire, then the condition in (1.6) that $a_{k} \geqq 0$ for all $k \geqq 2$, although sufficient for the $\mathscr{G}_{\text {_ }}$ inequalities, is too restrictive. For example, one can have $a_{2}>0, a_{3}<0, a_{4}>0$, and still satisfy the hypotheses of Theorem 1.2. On the other hand, we clearly need $a_{2}>0$ in order to have $V^{\prime \prime \prime}(x) \geqq 0$ for small $x$. We refer the reader to [27, Appendix], where the question of allowable negative coefficients is studied more closely. Concerning polynomial field theories of degree greater than four, we note that $H_{2 k}^{\prime \prime \prime}(x)$ does not have constant sign in $[0, \infty)$ for any $k>2$. Here, $H_{2 k}$ is the $2 k^{\prime}$ th Hermite polynomial $\exp \left(x^{2} / 2\right)(d / d x)^{2 k}$ $\cdot \exp \left(-x^{2} / 2\right)$. This is consistent with an observation of Spencer [26, p. 335].

\section{Appendix A. GKS Inequalities}

We give simple proofs of the first and second GKS inequalities

$$
\begin{aligned}
& \left(i_{1} \ldots i_{n}\right) \geqq 0, \\
& \left(i_{1} \ldots i_{n}\right)-\left(i_{1} \ldots i_{k}\right)\left(i_{k+1} \ldots i_{n}\right) \geqq 0, \quad k=1, \ldots, n-1,
\end{aligned}
$$

where $i_{1}, \ldots, i_{n} \in \Lambda(n \geqq 2)$ and $\varrho_{1}, \ldots, \varrho_{N}$ are arbitrary measures in $\mathscr{E}$.

Inequality (A.1) follows by expanding the exponentials in the representation

$$
\left(i_{1} \ldots i_{n}\right)=\left[Z\left(h_{1}, \ldots, h_{N}\right)\right]^{-1} E\left\{W_{i_{1}} \ldots W_{i_{n}} \exp \left(\sum J_{i j} W_{i} W_{j}\right) \exp \left(\sum h_{i} W_{i}\right)\right\},
$$


where $W_{i}, i=1, \ldots, N$, are independent random variables distributed by $\varrho_{i}$. For (A.2), we define $\boldsymbol{W}_{i}=\left(W_{i}^{(1)}, W_{i}^{(2)}\right)$ (where $W_{i}^{(\alpha)}, \alpha=1,2$, are two independent copies of $\left.W_{i}\right), \boldsymbol{h}_{i}=\left(h_{i}^{(1)}, h_{i}^{(2)}\right)$,

$$
\mathscr{D}=\partial^{n} / \partial h_{i_{1}}^{(2)} \ldots \partial h_{i_{n}}^{(2)}-\partial^{n} / \partial h_{i_{1}}^{(1)} \ldots \partial h_{i_{k}}^{(1)} \partial h_{i_{k}+1}^{(2)} \ldots \partial h_{i_{n}}^{(2)},
$$

and write the left-hand side of (A.2) as

$$
\left.\left[Z\left(h_{1}, \ldots, h_{N}\right)\right]^{-2} E\left\{\mathscr{D} \exp \left\{\sum \boldsymbol{h}_{i} \cdot \boldsymbol{W}_{i}\right\} \exp \left\{\sum J_{i j} \boldsymbol{W}_{i} \cdot \boldsymbol{W}_{j}\right\}\right\}\right|_{h_{i}^{(\alpha)}=h_{i}, \alpha=1,2} .
$$

We define $s_{i}=\left(s_{i}^{(1)}, s_{i}^{(2)}\right)=\tilde{A}^{t} \boldsymbol{h}_{i}$, where

$$
\tilde{A}=1 / \sqrt{2}\left(\begin{array}{rr}
1 & 1 \\
-1 & 1
\end{array}\right)
$$

$[\tilde{A}$ is the matrix used to define the matrix $A$ in (2.1). $] \mathscr{D}$ goes over to an operator $\mathscr{D}^{\prime}$, which is a sum of products of derivatives in the $s_{i}^{(\alpha)}$, each product appearing with a positive coefficient [17, Eq. (3.7)]. Inequality (A.2) follows from the fact that for any $\varrho_{i} \in \mathscr{E}$ the numbers $E\left\{\left(\tilde{A} \boldsymbol{W}_{i}\right)^{\boldsymbol{m}_{i}}\right\}, \boldsymbol{m}_{i}=\left(m_{i}^{(1)}, m_{i}^{(2)}\right)$, equal zero unless $m_{i}^{(1)}$ and $m_{i}^{(2)}$ are both zero, in which case this expectation is positive.

Other inequalities such as (1.11) are obtained by different choices of $\mathscr{D}^{\prime}$. Inequality (1.11) comes from

$$
\mathscr{D}^{\prime}=\partial^{2 n} / \partial s_{j_{1}}^{(2)} \ldots \partial s_{j_{2 n}}^{(2)} .
$$

\section{Appendix B. GKS Inequalities for Certain Non-Even Ferromagnets}

We establish GKS-type inequalities for two systems not directly covered by the preceding analysis. System $I$ is defined as in (1.1) with Hamiltonian $H_{\mathrm{I}}$ given by

$$
\begin{aligned}
& H_{\mathrm{I}}\left(x_{1}, \ldots, x_{N}\right) \\
& =-\sum_{1 \leqq i \leqq j \leqq N} J_{i j}\left(a x_{i}^{2}-b x_{i}\right)\left(a x_{j}^{2}-b x_{j}\right)+b \sum_{1 \leqq i \leqq N} h_{i} x_{i}-\sum_{1 \leqq i \leqq N} \mu_{i} x_{i}^{2},
\end{aligned}
$$

where $J_{i j} \geqq 0, h_{i} \geqq 0, \mu_{i}$ are real $(i, j=1, \ldots, N), a$ and $b$ are real, and with

$$
\varrho_{i}(d x)=(1 / 3)(\delta(x-1)+\delta(x)+\delta(x+1)), \quad i=1, \ldots, N .
$$

This system has been used as a model for ternary mixtures [16]. System II is defined as in (1.1) with Hamiltonian $H_{\text {II }}$ given by

$$
\begin{aligned}
& H_{\text {II }}\left(x_{1}, \ldots, x_{N}\right) \\
& =-\sum_{1 \leqq i \leqq j \leqq N} J_{i j} x_{i} x_{j}-\sum_{1 \leqq i \leqq j \leqq N} \gamma_{i j}\left(a x_{i}^{2}-b\right)\left(a x_{j}^{2}-b\right)-\sum_{1 \leqq i \leqq N} h_{i} x_{i},
\end{aligned}
$$

where $J_{i j} \geqq 0, \gamma_{i j} \geqq 0, h_{i} \geqq 0(i, j=1, \ldots, N), a$ and $b$ are real, and with the $\varrho_{i}$ 's as in (B.2). System II has been used to model ternary mixtures [16] and $\mathrm{He}^{3}-\mathrm{He}^{4}$ mixtures [1]. By methods similar to those used below, inequalities for higher spin systems and more complicated Hamiltonian may be established.

Theorem B.1. a) For System I, if $a \geqq|b|$, then for any $i_{1}, \ldots, i_{n} \in \Lambda(n \geqq 2)$ and all $J_{i j} \geqq 0, h_{i} \geqq 0, \mu_{i}$ real,

$$
\begin{aligned}
& E\left\{\prod_{j=1}^{n}\left(a X_{i_{j}}^{2}-b X_{i_{j}}\right)\right\} \geqq 0, \\
& E\left\{\prod_{j=1}^{n}\left(a X_{i_{j}}^{2}-b X_{i_{j}}\right)\right\}-E\left\{\prod_{j=1}^{k}\left(a X_{i_{j}}^{2}-b X_{i_{j}}\right)\right\} E\left\{\prod_{j=k+1}^{n}\left(a X_{i_{j}}^{2}-b X_{i_{j}}\right)\right\} . \\
& \geqq 0, k=1, \ldots, n-1 .
\end{aligned}
$$


b) For System II, if $a \geqq 2 b$ and $a \geqq 0$, then for any $i_{1}, \ldots, i_{n} \in \Lambda(n \geqq 2)$ and all $J_{i j} \geqq 0, \gamma_{i j} \geqq 0, h_{i} \geqq 0$,

$$
\begin{aligned}
& E\left\{X_{i_{1}} \ldots X_{i_{n}}\right\} \geqq 0, \quad E\left\{X_{i_{1}} \ldots X_{i_{n}}\right\}-E\left\{X_{i_{1}} \ldots X_{i_{k}}\right\} E\left\{X_{i_{k+1}} \ldots X_{i_{n}}\right\} \\
& \geqq 0, k=1, \ldots, n-1 .
\end{aligned}
$$

To prove Part a), we rewrite the Hamiltonian $H_{\mathrm{I}}$ in terms of the variables $w_{i} \equiv a x_{i}^{2}-b x_{i}$, isolate the part with the same form as (1.2), and redefine the measures $\varrho_{i}$. We have

$$
H_{\mathrm{I}}\left(x_{1}, \ldots, x_{N}\right)=H_{\mathrm{I}}^{\prime}\left(w_{1}, \ldots, w_{N}\right)+a \sum_{1 \leqq i \leqq N} h_{i} x_{i}^{2}-\sum_{1 \leqq i \leqq N} \mu_{i} x_{i}^{2},
$$

where

$$
H_{\mathrm{I}}^{\prime}\left(w_{1}, \ldots, w_{N}\right)=-\sum_{1 \leqq i \leqq j \leqq N} J_{i j} w_{i} w_{j}-\sum_{1 \leqq i \leqq N} h_{i} w_{i} .
$$

Hence, if we define

$$
\varrho_{i}^{\prime}(d w)=\left(\delta(w-(a-b)) e^{-a h_{i}+\mu_{\imath}}+\delta(w)+\delta(w-(a+b)) e^{-a h_{i}+\mu_{i}} /\left(1+2 e^{-a h_{2}+\mu_{i}}\right),\right.
$$

then the systems $\left\{H_{\mathrm{I}} ; \varrho_{i}, i=1, \ldots, N\right\}$ and $\left\{H_{\mathrm{I}}^{\prime} ; \varrho_{i}^{\prime}, i=1, \ldots, N\right\}$ are equivalent. Although the $\varrho_{i}^{\prime}$ are no longer even measures, the technique of Appendix A is still valid, and (B.4) follows once we prove that

$$
E\left\{\left(\tilde{A} \boldsymbol{W}_{i}\right)^{\boldsymbol{m}}\right\} \geqq 0 \quad \text { for all } \quad \boldsymbol{m}=\left(m^{(1)}, m^{(2)}\right) \geqq 0 .
$$

In (B.6), $\boldsymbol{W}_{i}=\left(W_{i}^{(1)}, W_{i}^{(2)}\right)$, where the $W_{i}^{(\alpha)}$ are two independent copies of a random variable distributed by $\varrho_{i}^{\prime}$. A straightforward calculation yields (B.6) provided $a \geqq|b|$.

Concerning Part b), we let $\boldsymbol{W}_{i}=\left(W_{i}^{(1)}, W_{i}^{(2)}\right)$, where $W_{i}^{(\alpha)}$ are two independent copies of a random variable distributed by $\varrho_{i}$, and

$$
Z_{i}=\left(a\left(W_{i}^{(1)}\right)^{2}-b, a\left(W_{i}^{(2)}\right)^{2}-b\right) .
$$

A check of Appendix A shows that it suffices to prove that

$$
\begin{aligned}
& E\left\{\left(\tilde{A} \boldsymbol{W}_{i}\right)^{\boldsymbol{m}}\left(\tilde{A} \boldsymbol{Z}_{i}\right)^{\boldsymbol{n}}\right\} \geqq 0 \text { for all } \boldsymbol{m}=\left(m^{(1)}, m^{(2)}\right) \geqq 0 \\
& \text { and all } \boldsymbol{n}=\left(n^{(1)}, n^{(2)}\right) \geqq 0 .
\end{aligned}
$$

A straightforward calculation yields (B.7) provided $a \geqq 2 b, a \geqq 0$. This completes the proof.

\section{References}

1. Blume, M., Emery, V.J., Griffiths, R. B.: Ising model for the $\lambda$ transition and phase separation in $\mathrm{He}^{3}-\mathrm{He}^{4}$ mixtures. Phys. Rev. A 4, 1071-1077 (1971)

2. Ellis, R. S.: Concavity of magnetization for a class of even ferromagnets. Bull A.M.S. 81, 925-929 (1975)

3. Ellis, R. S., Monroe, J. L.: A simple proof of the GHS and further inequalities. Commun. math. Phys. 41, 33-38 (1975)

4. Ginibre, J.: General formulation of Griffiths inequalities. Commun. math. Phys. 16, 310-328 (1970)

5. Glimm, J., Jaffe, A.: Absolute bounds on vertices and couplings. Rockefeller Univ. and Harvard Univ. (preprint) (1974)

6. Glimm,J., Jaffe,A., Spencer, T.: The particle structure of the weakly coupled $P(\varphi)_{2}$ model and other applications of high temperature expansions. In: Velo, G., Wightman, A.S. (Eds.): Constructive quantum field theory, p. 133-198. Berlin-Heidelberg-New York: Springer 1973 
7. Glimm,J., Jaffe, A., Spencer, T.: The Wightman axioms and particle structure in the $P(\varphi)_{2}$ quantum field model. Ann. Math. 100, 585-632 (1974)

8. Griffiths, R. B.: Correlation in Ising ferromagnets. J. Math. Phys. 8, 478-483 (1967)

9. Griffiths, R. B.: Rigorous results for Ising ferromagnets of arbitrary spins. J. Math. Phys. 10, $1559-1565$ (1969)

10. Griffiths, R. B.: Thermodynamics near the two-fluid critical mixing point in $\mathrm{He}^{3}-\mathrm{He}^{4}$. Phys. Rev. Letters 24, 715-717 (1970)

11. Griffiths, R.B., Hurst,C.A., Sherman, S.: Concavity of magnetization of an Ising ferromagnet in a positive external field. J. Math. Phys. 11, 790-795 (1970)

12. Griffiths, R. B., Simon, B.: The $\left(\varphi^{4}\right)_{2}$ field theory as a classical Ising model. Commun. math. Phys. 33, $145-164$ (1973)

13. Kelley, D., Sherman, S.: General Griffiths inequalities on correlations in Ising ferromagnets. J. Math. Phys. 9, 466-484 (1968)

14. Lebowitz, J. L.: Bounds on the correlations and analyticity properties of ferromagnetic Ising spin systems. Commun. math. Phys. 28, 313-321 (1972)

15. Lebowitz, J.L.: GHS and other inequalities. Commun. math. Phys. 35, 87 $\rightarrow 92$ (1974)

16. Mukamel,D., Blume, M.: Ising models for tricritical points in ternary mixtures. Phys. Rev. A 10, 610-617 (1974)

17. Monroe, J.L., Siegert, A.J.F.: GKS inequalities for arbitrary spin Ising ferromagnets. J. Stat. Phys. 10, 237-244 (1974)

18. Newman, C. M.: Gaussian correlation inequalities for ferromagnets. Z. f. Wahrscheinlichkeitsth. (to appear)

19. Newman, C. M.: Inequalities for Ising models and field theories which obey the Lee-Yang theorem. Commun. math. Phys. 41, 1-9 (1975)

20. Newman, C.M.: Zeroes of the partition function for generalized Ising systems. Comm. Pure Appl. Math. 27, 143-159 (1974)

21. Newman, C.M.: Moment inequalities for ferromagnetic Gibbs distributions. J. Math. Phys. (to appear)

22. Percus, J.: Correlation inequalities for Ising spin lattices. Commun. math. Phys. 40, 283-308 (1975)

23. Preston, C.: An application of the GHS inequalities to show the absence of phase transitions for Ising spin systems. Commun. math. Phys. 35, 253-255 (1974)

24. Simon, B.: Approximation of Feynman integrals and Markov fields by spin systems. Proc. of Internatl. Congr. of Mathematicians (Vancouver, B.C., 1974)

25. Simon, B.: Bose quantum field theory as an Ising ferromagnet: recent developments. Princeton Univ. (preprint) (1975)

26. Simon, B.: The $P(\varphi)_{2}$ Euclidean (quantum) field theory. Princeton, N.J.: Princeton University Press 1974

27. Sylvester, G.: Continuous-spin inequalities for Ising ferromagnets. M.I.T. (preprint) (1975)

28. Sylvester, G.: Private communication

29. Sylvester, G.: Representations and inequalities for Ising model Ursell functions. M.I.T. (preprint) (1974)

30. Thompson,C.: Mathematical statistical mechanics. New York: Macmillan 1972

Communicated by G. Gallavotti

Received August 3, 1975 